\title{
Performance of Turbo-Codes on Nakagami Flat Fading (Radio) Transmission Channels
}

\author{
Horia Balta ${ }^{1}$, Maria Kovaci ${ }^{2}$ and Alexandre de Baynast ${ }^{3}$ \\ ${ }^{1,2}$ Department of Communications, \\ University "Politehnica"of Timişoara, Faculty of Electronics and Telecommunications, \\ Postal address, 30223 Timişoara, Romania, \\ ${ }^{3}$ Department of Electrical and Computer Engineering, Rice University, MS-366 - 6100 Main Street \\ Houston, Texas 77005, E-Mail: horia.balta@etc.utt.ro,maria.kovaci@etc.utt.ro, debaynas@rice.edu
}

Technical Area: Communications Systems and Networking: 1. Error Detection and Correction 
Abstract: The performance in terms of Bit Error Rate and Frame Error Rate for a turbo-coded system in Nakagami frequency-nonselective (flat) fading multipaths channels are presented. An efficient method for generating Nakagami- $m$ fading samples is proposed. Through the parameter $m$, the Nakagami distribution can model signal fading conditions that range from severe to moderate. The primary justification for the use of the Nakagami- $m$ is its good fit to empirical fading data. By simulations, we show that Turbo-Code provide gain of tens of decidels over uncoded transmission in presence of Nakagami fading.

\section{INTRODUCTION}

The fading phenomenon occurs in radio transmission channels. It is due to the presence of multipaths that varies during the transmission, [1]. Radio channel modeling still remains a challenging issue. A sufficient and acceptable model is to consider the input-output relation of the digital channel of the form:

$$
y_{k}=\alpha_{k} \cdot x_{k}+w_{k},
$$

where $x_{k}$ and $y_{k}$ are the transmitted and received data for the time slot $k$, respectively; the parameter $\alpha_{k}$ is a random value which is characterized the time fluctuations from symbol to symbol (fast fading) or from block to block (block fading). Its distribution determines the channel type: Rayleigh, Rice or Nakagami. The input sequence $\left\{x_{k}\right\}$ is binary, random, in NRZ bipolar format, i.e. with unitary variance. Finally, the samples $w_{k}$ are zero-mean i.i.d. Gaussian variables of variance $\sigma^{2}$.

Shown in Fig. 1, the approach considered in this paper consists of two parts: the generation of the channel model and the BER computation.

In order to generate the transmitted sequence $\left\{x_{k}\right\}$ another sequence denoted $\left\{u_{k}\right\}$ is generated by random numbers with uniform distribution in the interval $[0,1)$. The targeted antipodal sequence is obtained after the following transformation:

$$
x_{k}=2 \cdot\left[2 \cdot u_{k}\right]-1
$$

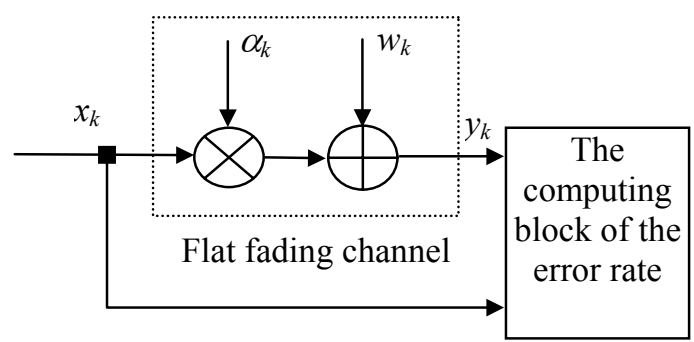

Fig. 1 Model considered through this paper in order to simulate the performance of a turbo-coded transmission over non frequency selective time-varying channels.

where [ ] denotes the truncated operation at the integer part. It can be shown that [1]:

$$
\overline{x_{k}^{2}}=1
$$

The sequence $\left\{\alpha_{k}\right\}$ follows a Nakagami distribution. By normalizing $\alpha_{k}$ such that $\overline{\alpha_{k}^{2}}=1$, the effective signal to noise ratio $E_{b} / N_{0}$ of the transmission is defined as follows [2]:

$$
\frac{E_{b}}{N_{o}}=\frac{1}{2} \cdot \frac{2 \cdot E_{b}}{N_{o}}=\frac{1}{2} \cdot \frac{\overline{\alpha_{k}^{2}} \cdot \overline{x_{k}^{2}}}{\overline{w_{k}^{2}}}=\frac{1}{2} \cdot \frac{1}{w_{k}^{2}},
$$

where $E_{b} / N_{0}=S N R(d B)$. Therefore, the variance of the additive noise can be expressed as:

$$
\overline{w_{k}^{2}}=\frac{1}{2 \cdot 10^{S N R / 10}}
$$

\section{THE NAKAGAMI DITRIBUTION}

The Nakagami- $m$ distribution has gained widespread application in the modeling of physical fading radio channels [3]. Through the parameter $m$, the Nakagami distribution can model signal fading conditions that range from severe to moderate. The primary justification for the use of the Nakagami- $m$ is its good fit to empirical fading data. Much theoretical and numerical analysis of the performances of diverse communication systems operating in Nakagami fading has been reported in the litterature. See for instance [3] and references therein.

Fewer results related to the computer simulation of Nakagami- $m$ fading have been reported.

In this paper, we give a procedure for simulating a Nakagami- $m$ fading channel, for arbitrary values of the fading parameter $m$. In addition, we derive a new useful approximation to the inverse cumulative distribution function of the Nakagami distribution, valid for arbitrary values of fading parameter $m$.

In this section, we briefly recall some basic properties of the Nakagami probability density function (pdf). We will refer to them in the next section in which we describe our random number generator.

The Nakagami- $m$ pdf is given by:

$$
p_{N}(\alpha)=\frac{2 \cdot m^{m}}{\Gamma(m)} \cdot \frac{\alpha^{2 m-1}}{\left(2 \sigma^{2}\right)^{m}} \cdot \exp \left(-m \frac{\alpha^{2}}{2 \sigma^{2}}\right)
$$

where $m \geq 1 / 2$ represents the fading figure.

The mean, $\bar{\alpha}$, and the value of the mean square, $\overline{\alpha^{2}}$, for $m$ $\geq 1$, integer, are the following:

$$
\bar{\alpha}=\sqrt{2 \sigma^{2}} \cdot \sqrt{\frac{\pi}{4 m}} \cdot P, \quad \overline{\alpha^{2}}=2 \sigma^{2}
$$


where $P=1$ for $\mathrm{m}=1$ and $P=\prod_{i=1}^{m-1} \frac{2 i+1}{2 i}$ for $m \geq 2$.

We assume in the sequel that $\overline{\alpha^{2}}=1$. It results that: $2 \sigma^{2}=1$ and $\bar{\alpha}=\sqrt{\pi / 4 m} \cdot P$. The function $\Gamma(m)$ is the wellknown gamma function given by the relation [1]:

$$
\Gamma(m)=\int_{0}^{\infty} t^{m-1} \cdot e^{-t} \cdot d t
$$

The generation of a random number with Nakagami distribution is done by the following change of variable:

$$
x=m \frac{\alpha^{2}}{2 \sigma^{2}}=m \alpha^{2}
$$

By replacing (9) in (6), the Nakagami probability density becomes:

$$
p_{x}(x)=\frac{p_{N}(\alpha)}{\frac{d x}{d \alpha}}=\frac{x^{m-1} \cdot e^{-x}}{\Gamma(m)} .
$$

The cumulative distribution function (cdf) of the random variable probability $x$ is obtained by the integration of the relation (10):

$$
F_{x}(x)=\int_{0}^{x} p_{x}(t) \cdot d t=\frac{1}{\Gamma(m)} \cdot \int_{0}^{x} t^{m-1} \cdot e^{-t} \cdot d t=\frac{\Gamma_{x}(m)}{\Gamma(m)} .
$$

If $m$ is a natural number, then:

$$
F_{x}(x)=1-\sum_{k=0}^{m-1} \frac{x^{k}}{k !} \cdot e^{-x}
$$

$F(x)$ has an inflexion point $x_{F}$ obtained by zeroing its second order derivative:

$$
\begin{aligned}
& \frac{d^{2} F_{x}(x)}{d x^{2}}=\frac{d p_{x}(x)}{d x}= \\
& =\frac{1}{\Gamma(m)} \cdot\left[(m-1) \cdot x^{m-2}-x^{m-1}\right] \cdot e^{-x}, \quad x \geq 0, \quad m \neq 1,
\end{aligned}
$$

where:

$$
x_{F}=m-1 \text {. }
$$

\section{THE RANDOM NUMBERS GENERATION WITH NAKAGAMI DISTRIBUTION}

After recalling the basic properties of the Nakagami pdf, we propose in this section a new random number generator for Nakagami distribution.

It is well known that transforming a uniform random variable $u$ which belongs to the interval $[0,1)$ by the inverse cdf $F_{x}^{-1}(u)$ results in a transformed random variable having a cdf given by $F_{x}(x)$ [3]. Thus $F_{x}^{-1}(u)$ is a random variable having Nakagami- $m$ distribution. In order to implement a direct method to generate Nakagami- $m$ distributed random variates, the Nakagami- $m$ inverse cdf is needed. Since this is not available in closed-form, $F_{x}^{-1}(u)$ can only be approximated numerically. An algorithm based on the fixed-point method, with $u \in[0,1)$, has been proposed in [4]. Here, we briefly recall the main steps:

Step 1. $u \in[0,1)$ is generated following the uniform distribution

Step 2. $x_{F}, p_{x}\left(x_{F}\right)$ and $F_{x}\left(x_{F}\right)$ are computed from (14), (10) and (11) with $m$ known;

Step 3. $x_{l}$ is computed with the relation:

$$
\left(x_{1}-x_{F}\right) \cdot p_{x}\left(x_{F}\right)=u-F_{x}\left(x_{F}\right),
$$

Or equivalently:

$$
x_{I}=x_{F}+\frac{u-F_{x}\left(x_{F}\right)}{p_{x}\left(x_{F}\right)} ;
$$

Step 4. $F_{x}\left(x_{l}\right)$ is computed which is compared to $u$. If:

$$
\left|F_{x}\left(x_{1}\right)-u\right|<\varepsilon
$$

where $\varepsilon$ is the targeted precision of the approximation. If the relation (17) is satisfied, the algorithm is stopped. Otherwise, steps 3 and 4 are repeated, updating $x_{1}$ with $x_{2}$ and $x_{F}$ with $x_{1}$.

In Fig. 2, we illustrate the generating process described above through an example. We considered the values: $m=2$, $u=0.8, \quad \varepsilon=10^{-5}$; we obtained successively: $x_{F}=1.0$, $x_{1}=2.456344, x_{2}=2.913904, x_{3}=2.992212, x_{4}=2.994307$, $F_{x}\left(x_{3}\right)=0.8$.

Obviously, the $x_{k}$ string is convergence for a finite number of iterations toward $F_{x}^{-1}(u)$ regardless of the sign of the quantity $u-F_{x}\left(x_{F}\right)$.

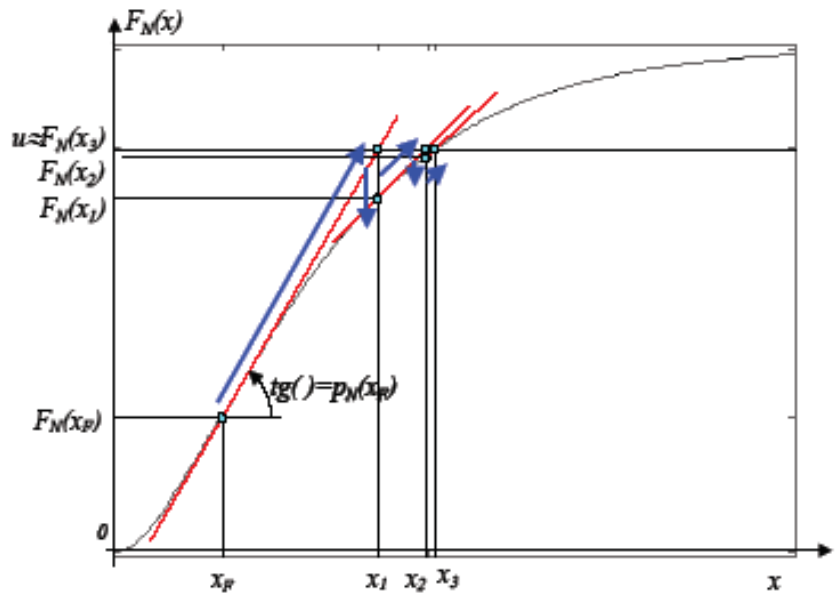

Fig.2 Illustration of the proposed random generator with Nakagami distribution.

Remark: The proposed algorithm can be used for any value of $m$. However, in case of $m$ is not natural, the computation of two integrals for the values $\Gamma_{x}(m)$ and $\Gamma(m)$ are required. 


\section{THE TURBO-CODED TRANSMISSION SYSTEM IN NAKAGAMI FLAT FADING}

The Turbo-Coded (TC) scheme that we considered in our simulations is presented in Fig.3.

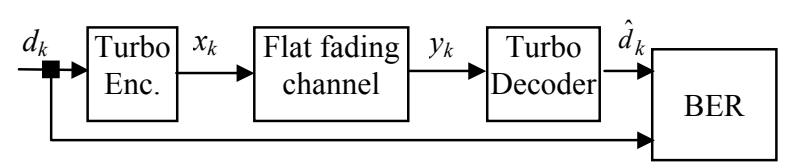

Fig. 3 The turbo-coded transmission system through flat fading channel.

The component decoders use a MAP or SOVA iterative algorithm. The different steps of the MAP decoding algorithm are summarized in Fig. 4 [5].

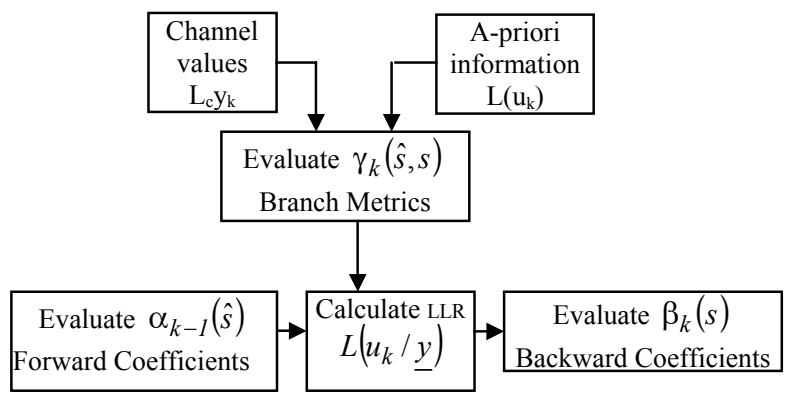

Fig. 4. Summary of the key operations in the MAP algorithm.

To decoding the received sequences from flat fading channel (whit his input-output relation done by (1), where $\alpha$ is done by (7)), the MAP algorithm must be adapted to $L_{c}$ coefficients (channel reliability), so:

$$
L_{c}=4 \cdot R \cdot B \cdot \bar{\alpha}
$$

where $B$ is the absolute value of the SNR, $R$ is the turbocoding rate and $\bar{\alpha}$ depends of $m$. If $m=1, \bar{\alpha}=\sqrt{\frac{\pi}{4}}=0.8862$, [6].

\section{EXPERIMENTAL RESULTS}

We considered the following setup for our simulations. A Recursive Systematic Convolutional Code, $1 / 3$ rate is employed, [7]. A S-interleaver is used. The simulations are made for BPSK signaling. A stop criterion after each iteration is applied when the decoded sequence matches with a codeword. However, a maximal number of 15 iterations is tolerated. The transmission channel is the Nakagami flat fading channel described in the previous sections.

The performance of TC for Nakagami flat fading channels, with $m>1$ are upper bounded by the Rayleigh flat fading performances (which correspond to the Nakagami flat fading channel with $m=1$ ) and lower bounded by static channels (which correspond to the Nakagami flat fading channel with $m=\infty$ ).

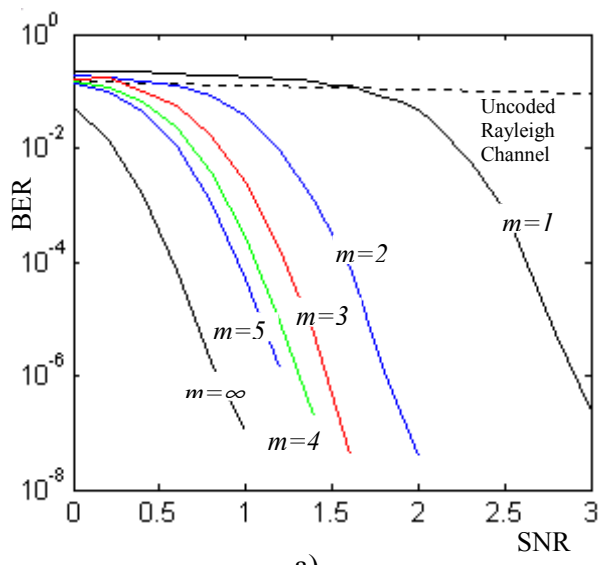

a)

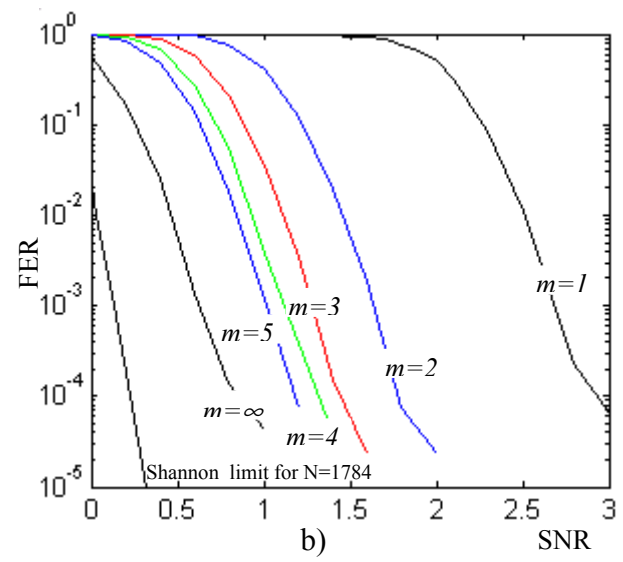

Fig. 5 Performance for Turbo-Coded transmission over flat fading Nakagami channel - a) The Bit Error rate (BER) is plotted as a function of the SNR for various fading parameters $m$ described in Eq.6. b) Similarly, the Frame Error rate (FER) is plotted as a function of the SNR.

\section{CONCLUSIONS}

In this paper the BER (Bit Error Rate) and FER (Frame Error Rate) performances obtained from the function simulation of the turbo-coded transmission systems in the Nakagami frequency-nonselective (flat) fading multi-paths channels are presented.

From the curves of Fig.5 results that the channel models with a Nakagami flat fading cover a large scale of practice situations. The simulations presented in this paper prove that using the $\mathrm{TC}$ in the presence of the fading provides a huge performance gain of the order of tens of decibels.

The random number generation with Nakagami distribution presented in Sec. III, can be used in more sophisticated turbo-coded transmissions including higher order modulation, OFDM transmission and multiple transmit/receive antennas. 


\section{REFERENCES}

[1] John G. Proakis, "Digital communications", McGraw-Hill Series in Electrical and Computer Engineering Stephen W., 2001,

[2] Li-Der Jeng, $\mathrm{Yu}$ T. Su and Jung-Tang Chiang, "Performance of Turbo Codes in Multipath Fading Channels", VTC'98, pp. 61-65,

[3] N. Beaulieu, C. Cheng, "Efficient Nakagami-m Fading Channel Simulation", IEEE journal on Vehicular Technology, vol. 54, no. 2, March 2005,

[4] M. C. Jeruchim, P. Balaban, K.S. Shanmugan, ,Simulation of Communication Systems, Modeling, Methodology and Techniques", second edition, Kluwer Academic, 2000,

[5] L.Hanzo, T.H.Liew, B.L.Yeap, "Turbo Coding, Turbo Equalisation and Space-Time Coding for Transmission over Fading Channels", John Wiley \& Sons Ltd, England, 2002,

[6] Eric K. Hall, Stephen G. Wilson, Member, IEEE, "Design and Analysis of Turbo Codes on Rayleigh Fading Channels", IEEE journal on selected areas in communications, vol. 16, no. 2, February 1998 ,

[7] C. Berrou, A. Glavieux, P. Thitimajshima - "Near Shannon limit error-correcting coding and decoding: Turbo-codes", Proc.ICC'93, Geneva, Switzerland, May 1993, pp. 1064 1070. 